\title{
Multi-Objective Optimal Kinematic Design of 3-TPS/TP Parallel Robot Manipulator
}

\author{
Jiman Luo 1,2, a , Dalong Wang ${ }^{2, b}$, Zhihui Xing ${ }^{1, c}$, Ngai Ming Kwok ${ }^{2, d}$ \\ ${ }^{1}$ School of Traffic \& Mechanical Engineering, Shenyang Jianzhu University, Shenyang, China \\ ${ }^{2}$ School of Mechanical \& Manufacturing Engineering, University of New South Wales, Australia

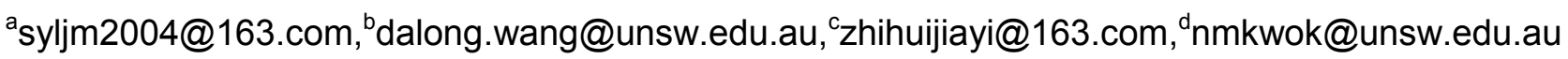

Keywords: Multi-objective Optimization, Parallel Robot Manipulator, Particle Swarm Optimization

\begin{abstract}
The purpose of this research work is to find the optimized kinematic structural parameters of the 3-TPS/TP parallel manipulator while taking into consideration the workspace, kinematic properties and other factors. The Ranked Pareto Particle Swarm Optimization (RP-PSO) approach is applied to solve the multi-objective optimization problems. Firstly, the structure of the parallel manipulator and its kinematic model are devised. Then, we set up the kinematical performance indices for the evaluation of manipulator working properties, including Local Condition Index (LCI), Global Condition Index (GCI) and Good Condition Workspace (GCW). The optimization is further carried out in a Parameter Design Space (PDS) with normalized geometry parameters. Then the geometry optimization problems are solved using a PDS based RP-PSO approach. Finally, the feasibility of this approach is supported by examples given this paper.
\end{abstract}

\section{Introduction}

The parallel robot manipulators (PRM) have been actively researched for the following advantages: simple and compact structure, higher speed, higher stiffness, large carrying capacity and high machining accuracy [1]. In the family of these parallel robots, many 3-DOF parallel robots have been developed [2]. One example is planar 3-RRR (Revolute-Revolute-Revolute) PRM [3]. The moving platform has three planar DOFs, which are two translations in a plane and rotation around the axis perpendicular to the plane. Another example is the 3-PTT (Prismatic-Hooke-Hooke) PRM, which has only 3 translational DOF along $x, y$ and $z$ axis [4]. One of the parallel robots presented by Hunt is the 3-RPS (Revolute-Prismatic-Spherical) parallel robot with 3 chains. Each chain consists of a revolute joint, a prismatic joint and a spherical joint, respectively [5]. Another PRM with 3 translational DOFs is the Star, designed by Hervé based on group theory [6].

This paper focuses on the optimal kinematic design of a new 3-TPS/TP PRM shown in Fig. 1. The moving platform of the new PRM is able to achieve three-dimensional motion through combination of two rotational movements and one translational movement [7].There are many performance indicators available to describe a PRM's motion traits, e.g. the manipulability and workspace size. These indicators are often conflicting in real applications. For example, maximizing the workspace volume often tends to produce singularity in manipulator configurations, while considering only manipulability may lead to architectures with relatively small workspaces [8]. Thus a multi-objective optimization approach for PRM design is required.

Some other methods have been presented. In these approaches, an objective function is defined based on specific requirements; numerical methods are then utilized to obtain optimal results. Stock et al. proposed a method using search minimization to optimize kinematic design of linear Delta robot [9]. Zhang et al. applied genetic algorithms for the multiple-criteria kinematic optimization of spherical serial mechanisms [10]. Stan et al. presented the optimal design of 3- DOF DELTA linear translation parallel robot also based on genetic algorithm approach [11].

The purpose of this research work is the search for the optimized kinematic structural parameters of a new PRM while balancing between the working space and kinematic properties. The ranked Pareto particle swarm optimization (RP-PSO) approach is adopted due to its fast convergence [12]. 


\section{The structure and kinematic models of the parallel manipulator}

The structure of the PRM. The structure of 3-TPS/TP robot manipulator to be studied in this paper is shown in Fig. 1. The kinematic diagram is given in Fig. 2. The regular triangle denoted by $B_{1} B_{2} B_{3}$ is a fixed platform and $R_{1}$ is the radius of its circumcircle. The moving platform is denoted by regular triangle $\mathrm{b}_{1} \mathrm{~b}_{2} \mathrm{~b}_{3}$. $R_{2}$ is the radius of its circumcircle. These two platforms are connected by three links, which are $B_{1} C_{1} b_{1}, B_{2} C_{2} b_{2}$ and $B_{3} C_{3} b_{3}$ respectively. Please note that $B_{i}(i=1,2,3)$ are the centers of the Hookes joints, $b_{i}(i=1,2,3)$ are the centers of the spherical joints, $C_{i}(i=1,2,3)$ are the centers of the Prismatic joints. The fourth link, $A_{1} C_{4} A_{2}$, connects the center of the fixed platform $\left(A_{1}\right)$ and moving platform $\left(A_{2}\right)$, where $A_{1}$ is Hooke joint and $C_{4}$ is a prismatic joint.

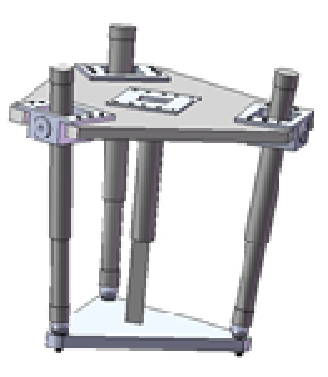

Fig. 1. The structure of PRM

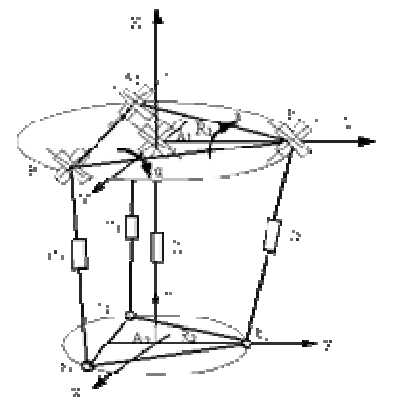

Fig. 2. The kinematic diagram of PRM

A global coordinate system $\mathrm{A}_{1}-\mathrm{XYZ}$ is attached to the center of the fixing platform, and a moving coordinate system $\mathrm{A}_{2}$-xyz is attached to the center of the moving platform. The $\mathrm{z}$-axis passes through the center of the moving platform and is perpendicular to the equilateral triangle $b_{1} b_{2} b_{3}$. The $\mathrm{y}$-axis and $\mathrm{Y}$-axis pass through the center of moving platform and fixing platform, respectively, and are along the perpendicular lines of equilateral triangles. The initial distance between the moving platform and fixed platform is denoted by $H$. The movement of moving platform can be described by rotation angle $\alpha, \beta$ along $\mathrm{X}$ and $\mathrm{Y}$ axis, and extended along $\mathrm{Z}$ axis.

Coordinate Transformation Equations. In the moving coordinate system, the coordinates of each hinge point $b_{i}$ can be described by rotation around $\mathrm{X}$ axis with angle $\alpha$, and around $\mathrm{Y}$ axis with angle $\beta$, and displacement $\Delta Z$ in the $\mathrm{Z}$ direction. The coordinate transformation equations from the moving coordinates to the global coordinates are given by:

$$
\left(X_{b i}, Y_{b i}, Z_{b i}, 1\right)^{T}=\left[T_{o p}\right]\left(x_{b i}, y_{b i}, z_{b i}, l\right)^{T}
$$

where $\left[T_{o p}\right]=\left[\begin{array}{cc}R & P \\ 0 & 1\end{array}\right], R=\left[\begin{array}{ccc}\cos \beta & \sin \beta \sin \alpha & \sin \beta \cos \alpha \\ 0 & \cos \alpha & -\sin \alpha \\ -\sin \beta & \cos \beta \sin \alpha & \cos \beta \cos \alpha\end{array}\right], P=\left[0,0, Z_{P}\right]^{T}, Z_{p}=H+\Delta Z$

The Jacobian matrix can then be derived from coordinate transformation equation.

Jacobian matrix. The inverse kinematics equation can be used to solve the elongation lengths of the links when the locations of points are known. That is

$$
\begin{aligned}
& L_{l}=\sqrt{\left(X_{B I}-x_{b 1} \cos \beta-y_{b l} \sin \alpha \sin \beta\right)^{2}+\left(Y_{B I}-y_{b l} \cos \alpha\right)^{2}+\left(x_{b l} \sin \beta+y_{b l} \cos \beta \sin \alpha+z_{p}\right)^{2}} \\
& L_{2}=\sqrt{\left(y_{b i} \sin \beta \sin \alpha\right)^{2}+\left(Y_{B 2}-y_{b i} \cos \alpha\right)^{2}+\left(y_{b i} \cos \beta \sin \alpha+z_{p}\right)^{2}} \\
& L_{3}=\sqrt{\left(X_{B 2}-x_{b 3} \cos \beta-y_{b 3} \sin \alpha \sin \beta\right)^{2}+\left(Y_{B 3}-y_{b 3} \cos \alpha\right)^{2}+\left(x_{b 3} \sin \beta+y_{b 3} \cos \beta \sin \alpha+z_{p}\right)}
\end{aligned}
$$

The Jacobian matrix which reflects the relationship between the speed of elongation of links and the velocity of center point of moving platform is defined as:

$$
\mathbf{v}_{\mathbf{p}}=\mathbf{J} \mathbf{v}_{\mathbf{l}}=\mathbf{T}^{-1} \mathbf{v}_{\mathbf{l}}
$$

where $\mathbf{v}_{\mathbf{p}}$ is the velocity of center point in moving platform, $\mathbf{v}_{\mathbf{l}}$ is the speed of elongation of legs, $\mathbf{J}$ is Jacobian matrix, $\mathbf{T}$ is inverse Jacobian matrix. 


\section{The kinematical performance indices}

In this research, we aim to optimize the manipulator's geometry to obtain high flexibility and large workspace. This section introduces several kinematical performance indices for the evaluation of manipulator's working property.

Parameter Design Space (PDS). The parameter design space is a useful concept to normalize geometry parameters introduced in [8]. There are three structure parameters $R_{1}, R_{2}$ and $H$ in the 3-TPS/TP parallel manipulator, which are denoted as $L_{i}(i=1,2,3)$. As stated in [8], the parameter $D$ is defined as the sum of all characteristic parameters. Then, the non-dimensional parameters $l_{i}\left(r_{1}, r_{2}, h\right)$ can be obtained by the following equation:

$l_{i}=L_{i} / D$

For the manipulator studied here, all the three parameters $l_{i}$ can be considered as non-dimensional parameters. We have

$$
l_{1}+l_{2}+l_{3}=1,\left\{\begin{array}{c}
0<l_{1}, l_{2}, l_{3}<1 \\
l_{2}<l_{1}
\end{array}\right.
$$

Global Conditioning Index (GCI). The Global Conditioning Index (GCI) is defined as in [8]:

$$
\eta=\frac{\int_{w} \mu d w}{\int_{w} d w}
$$

where $\mathrm{W}$ is the workspace, $\mu$ is the local conditioning index (LCI) defined as the reciprocal of the condition number of the Jacobian matrix.

$$
\mu=1 /\|\mathrm{J}\| \cdot\left\|\mathrm{J}^{-1}\right\|
$$

A larger $\mu$ value means better dexterity. Thus the minimum LCI is usually an indicator of singularity occurrence. However, the value of LCI will vary with end-effecter locations and postures. The Global Conditioning Index (GCI) is proposed to provide a global assessment of the adverse effect of singularity. The GCI is the average of LCI over the whole workspace. The value of GCI only changes with different structural parameters. A larger $\eta$ usually indicates better kinematical performance in the entire workspace. GCI should be calculated only in the good-condition workspace (GCW), where the LCI values over the workspace are greater than or equal to the minimum LCI. The purpose of our optimization is to find geometry parameters which will bring a large work space with good condition. Therefore, our optimization objectives are to maximize GCI and GCW simultaneously.

\section{The multi-objective optimization by RP-PSO approach}

Formulation of the optimization problem. The aim of optimal kinematic design is to solve the multi-objective, non-linear optimization problem by RP-PSO approach, by way of selecting geometric design variables for the 3-TPS/TP PRM to provide an acceptable compromise between manipulability and workspace. The optimization is carried out in PDS with unified parameter. The goal is to maximize GCI and GCW simultaneously, that is,

$$
\max _{P \in \Phi} F(X), X \in R^{n}
$$

where $X=\left\{l_{1}, l_{2}\right\}$ is a 2-dimensional vector, and the parameter $l_{1}$ and $l_{2}$ is normalized geometry parameters in the PDS. $\phi$ defines a feasible set of $P$ values. $F=\left\{f_{1}, f_{2}\right\}$ is a vector with 2 objective components to be maximized. In this work, $f_{1}=\{\eta\}, f_{2}=\{W\}$. Here, $\eta$ refers to GCI in (6), W is the volume of GCW solved by integration. 
Ranked Pareto Particle Swarm Optimization. The Ranked Pareto Particle Swarm Optimization (RP-PSO) follows the basic idea of PSO but is capable of solving multi-objective optimization problems efficiently [14]. In the RP-PSO, potential solutions as particles are initiated randomly in the search space; these particles are then evaluated for their qualities with regard to all objectives. Those particles with highly-ranked qualities have preferences to enter the set of Global Best vectors, which stores many currently best solutions found by particles. Thus, particles in RP-PSO will search towards many possible directions and the diversity among solutions is well preserved. Ideally, a set of optimal solutions will be found when the termination criterion is met. The process using RP-PSO for PRM geometry optimization is detailed below.

Step 1: Initialize particles. At the beginning of iterations, particles are initialized randomly in the search space, which are denoted by $X_{i}\left(l_{1}, l_{2}\right), i=1,2, \ldots, P$, where $P$ is the number of particles.

Step 2: Calculate Raw Fitness Value (RFV) of each function for particles.

$$
R F V_{j, i}=f_{j}\left(X_{i}\right), j=1,2
$$

where $i$ is the particle number, $f_{1}\left(X_{i}\right)$ is the value of GCI denoted by $\eta, f_{2}\left(X_{i}\right)$ is the volume of GCW denoted by $W$. Then we get a raw fitness value matrix (RFVM), which is a $2 \times P$ array of raw fitness values of this generation.

Step 3: Transform RFVM to Modified Fitness Value (MFV) by comparing the RFV of each particle with those of other particles. The RFV of each particle is compared with RFV of all other particles. We define that particle $i_{1}$ is said to dominate particle $i_{2}$ at objective $j$ if

$$
R F V_{j, i 1}>R F V_{j, i 2}, j=1,2
$$

The MFV is defined to be the number of particles dominated by this particle at single objective. The larger RFV is, the greater the number of MFV is. MFV shows the qualities of particles regarding objective $f_{i}$, i.e., particles with higher MFV are more desirable than those particles with lower MFV.

Step 4: Particles are ranked according to their qualities with regard to all objectives. The sum of all MFVs is called to be a global modified fitness vector (GMFV), which is a $P$-dimensional vector:

$$
G M F V=\sum_{j=1,2} M F V V_{j}
$$

The GMFV can be treated as a benchmark of particle quality, in which particle quality with regard to all objectives are taken into account. Particles with higher GMFVs have higher aggregate qualities than those with lower GMFVs in general.

Step 5: Particle Rank Value (PRV) is rounded to integers Particle Copy Number (PCN), and arranged in descending order according to PCN. Here, Global Best in RP-PSO is a $P$-dimensional vector which stores $P$ best locations found by all particles. To do this, we first set a particle rank value, denoted by PRV, for each particle. The PRV of $i^{\text {th }}$ particle is defined by

$$
P R V_{i}=\frac{G M F V_{i}}{\sum_{j-1, \ldots, P} G M F V_{j}} \times P, i=1,2, \ldots, P
$$

The $P R V_{i}$ is then rounded to an integer denoted by Particle Copy Number (PCN), which determines how many copies of this particle enter the Global Best $\left(G_{b e s t}\right)$ of this generation:

$$
P C N_{i}=\operatorname{Round}\left(P R V_{i}\right), i=1,2, \ldots, P
$$


To ensure that there are enough candidates for $G_{b e s t}$, this function rounds to the nearest integer towards infinity. The particles which hold higher PRVs have the priority to enter the $G_{b e s t}$ vector. This process continues until the $G_{\text {best }}$ vector is filled up. Particles with lower PRVs, not contributing to solution improvements, will not enter the $G_{\text {best }}$ if it has been filled by copies of particles with higher PRVs and redundant particles will be abandoned.

Step 6: The best histories of particles are stored in a $P$-dimensional vector, denoted by $P_{b e s t}$.

Step 7: Update to create the new particle locations. Each particle updates its position according to the following equations.

$$
\begin{aligned}
& V_{\text {new }}=\varepsilon \times v_{\text {old }}+c_{1} \times \operatorname{rand}_{1}() \times\left(P_{\text {best }}-X_{\text {old }}\right)+c_{2} \times \operatorname{rand}_{2}() \times\left(G_{\text {best }}-X_{\text {old }}\right) \\
& X_{\text {new }}=X_{\text {old }}+V_{\text {new }}
\end{aligned}
$$

where $\varepsilon, c_{1}$ and $c_{2}$ are weighting factors. In RP-PSO, $G_{b e s t}$ is a $P$-dimensional vector which stores best $P$ positions found by particles. As a consequence, particles in RP-PSO will search towards many possible directions. In this way, the diversity among solutions is well preserved.

Step 8: The optimization process stops when the termination criteria are met, e.g. 50 iterations.

\section{Numerical example}

This section presents an example using RP-PSO approach for geometry optimization of parallel robot manipulator. The range of rotation angles including $\alpha$ and $\beta$ are set to be $\left[-20^{\circ}, 20^{\circ}\right]$. Suppose that the desired task workspace of the robot is $0.125 \mathrm{~m}^{3}$, the maximum range of movement along $\mathrm{z}$ in the passive chain is: $0<\Delta Z<0.3 \mathrm{~m}$. The optimization process is described in detail as below:

The structure parameters to be optimized are $R_{1}, R_{2}$ and $H$. Let $R_{1}=L_{1}, R_{2}=L_{2}, H=L_{3}$. Using (4) and (5), we set up a parameter design space consisting of $l_{1}$ and $l_{2}$. In this example, the particle number is set to $P=300$. The RP-PSO parameters $\varepsilon$ is a fraction with $\varepsilon \in\left[\begin{array}{ll}0 & 0.5\end{array}\right], c_{1}=2$, $c_{2}=2$. The iteration reaches a stable state at $G=50$.

The optimization results are shown in Fig. 3, in which the Pareto front is denoted by red stars. The blue dots show the positions of other particles at the end of iteration. The Pareto front consists of thirty nine particles. In Fig. 3, point $\mathrm{A}$ is the optimization result using the approach in our previous research [8]. It is clear that all particles on the Pareto front are Pareto Optimal and not dominated by any other solutions (including A) in the search space. For example, points B and C are particles on the Pareto front. The values of WSV and GCI of points A, B and C are listed in Table 1.

The WSV and GCI of A are 0.065 and 0.0807, respectively. The effectiveness of the proposed optimization method is explicitly noticeable from significant increases in both WSV and GCI. The WSV of point B is increase from 0.065 of point A to 0.0673 ; the GCI is increased from 0.0807 to 0.0877 . For point $C$, the WSV and GCI are increased to 0.0988 and 0.0815 , respectively.

Please note that all points on the Pareto front will fulfill our requirements. In this paper, we calculate the structure dimensions from the results of Point B. Starting from practical requirements, we choose $H=0.5 \mathrm{~m}$. Then, the normalization factor $D=H / l_{3}=1.278$, then, $R_{1}=l_{1} D=0.395 \mathrm{~m}$, $R_{2}=l_{2} D=0.382 \mathrm{~m}$. The volume of the optimized workspace is $0.1677 \mathrm{~m}^{3}$, which is large than the desired task workspace $\left(0.125 \mathrm{~m}^{3}\right)$.

Table 1 Optimization results

\begin{tabular}{ccccc}
\hline Particle & $\mathrm{L}_{1}$ & $\mathrm{~L}_{2}$ & WSV & GCI \\
\hline $\mathrm{A}$ & 0.3955 & 0.2227 & 0.065 & 0.0807 \\
$\mathrm{~B}$ & 0.3093 & 0.2994 & 0.0673 & 0.0877 \\
$\mathrm{C}$ & 0.2471 & 0.2443 & 0.0988 & 0.0815 \\
\hline
\end{tabular}




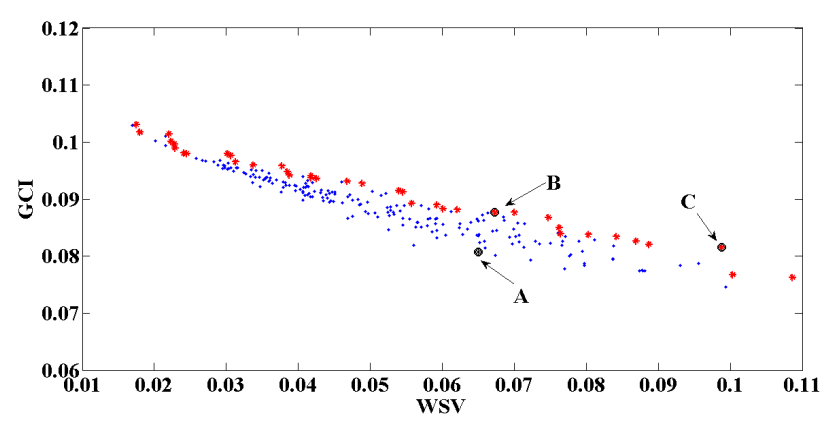

Fig. 3. Optimization results and Pareto front

\section{Conclusion}

In this paper, a new geometry optimization methodology is presented for kinematic design of the parallel manipulator. The optimization objectives are set to be maximizing the manipulability and workspace simultaneously. The RP-PSO approach is adopted to find the optimization parameters in normalized parameter space. The effectiveness of the methodology is verified by numerical studies on a 3-TPS/TP parallel manipulator.

\section{Acknowledgment}

This work has been supported by "Natural Science Foundation" of Liaoning Province (20102185) and "Open Foundation of Key Laboratory" of Liaoning Province (College) (JX-200911).

\section{References}

[1] J. Arata, H. Kondo, N. Ikedo and H. Fujimoto, Haptic device using a newly developed redundant parallel mechanism, IEEE Transactions on robotics, 27, 2 (2011) 201-214.

[2] F. Patanè and P. Cappa, A 3-DOF parallel robot with spherical motion for the rehabilitation and evaluation of balance performance, IEEE Transactions on neural systems and rehabilitation engineering, 19, 2 (2011) 157-166.

[3] C.M. Gosselin, S. Lemieux, J.-P. Merlet, A new architecture of planar three-degrees-offreedom parallel manipulator, Proceedings of the IEEE International Conference on Robotics and Automation, Minneapolis, MN, April 22-28, 1996, pp. 3738-3743.

[4] M. Hu, W. Wang; G. Cai, Development and study of a kind of parallel robot with 3-DOF, Proceedings of the 2008 Chinese Control and Decision Conference, pp. 2887-90, 2 July 2008.

[5] K.H. Hunt, Structural kinematics of in-parallel-actuated robot arms, Mechanical Transmission and Automation Design, 105 (1983) 705-712.

[6] J.M. Hervé, Group mathematics and parallel link mechanisms, Proceedings of the International Symposium on Robotics, Mechatronics, and Manufacturing Systems, 1992, pp. 459-464.

[7] J. Luo, D. Wang, Y. Jiang, Geometry optimization of a new hybrid robot manipulator, submitted to the 2011 IEEE International Conference on Mechatronics and Automation.

[8] X. J. Liu and J. Wang, A new methodology for optimal kinematic design of parallel mechanisms, Mechanism and Machine Theory, 42 (2007) 1210-1224.

[9] M. Stock and K. Miller, Optimal kinematic design of spatial parallel manipulators: application to linear Delta robot, Mechanical Design, 125 (2003) 292-301.

[10] X. Zhang, C. A. Nelson, Multiple-criteria kinematic optimization for the design of spherical serial mechanisms using genetic algorithms, Mechanical Design, 133(2011), 1-10.

[11] S. D. Stan,.V. Maties,R. Balan, C. Lapusan, Genetic algorithms to optimal design of a 3 DOF parallel robot, Proceedings of the 2008 IEEE International Conference on Automation, Quality and Testing, Robotics, pp. 365-370, 2008.

[12] D. Wang, N. M. Kwok, D. K. Liu and Q. P. Ha, Ranked pareto particle swarm optimization for mobile robot motion planning, Design and Control of Intelligent Robotic Systems, Berlin Heidelberg: Springer-Verlag, 2009, pp. 97-118. 